\title{
Ease-of-retrieval effects on procedural justice judgements under conditions of informational and personal uncertainty
}

\author{
Juan Liang, ${ }^{1}$ Hongyu $\mathrm{Ma},{ }^{1}$ Kees van den Bos ${ }^{2}$ Xiaorong Cheng, ${ }^{1}$ Bin Wang, ${ }^{1}$ Hengqing Tong ${ }^{3}$ \\ and Xucheng Guo ${ }^{1}$ \\ ${ }^{1}$ Central China Normal University, ${ }^{3}$ Wuhan University of Technology, Wuhan, China, and ${ }^{2}$ Utrecht Univesrsity, Utrecht, \\ Netherlands
}

\begin{abstract}
This study tests whether individuals' reliance on ease-of-retrieval processes when forming procedural justice judgements are moderated by informational and personal uncertainty. In Studies 1 and 2 we examined the predicted effects of informational uncertainty. Results indicated that participants in information-uncertain conditions relied on ease-of-retrieval, whereas those in information-certain conditions relied on content information to make procedural justice judgements. In Study 3 we examined the combined effects of informational uncertainty and personal uncertainty on reliance on ease-of-retrieval when forming procedural justice judgements. The findings of Study 3 indicated that personal uncertain participants who were in informational certain conditions based their procedural justice judgements on content information, whereas all other participants based their procedural justice judgements on ease-of-retrieval. This is the first paper to demonstrate that the joint effect of informational uncertainty and personal uncertainty on reliance on ease-of-retrieval is different from the two uncertainties acting alone.
\end{abstract}

Key words: ease-of-retrieval, informational uncertainty, personal uncertainty, procedural justice judgements.

\section{Introduction}

Procedural justice reflects the perceived fairness of decisionmaking processes (Van den Bos, 2001). Procedural justice judgements play an important role in human life. For example, high levels of procedural justice may affect support of authorities (e.g., Tyler, 2006) and lead to increased job satisfaction (e.g., Ambrose \& Schminke, 2009). Thus, it is important to examine the source of information on which people rely when forming procedural justice judgements. A

Correspondence: Hongyu Ma, School of Psychology, Central China Normal University, Wuhan 430079, Hubei, China. Email: mahy@ mail.ccnu.edu.cn

The research was supported by the Project of the National Natural Science Foundation of China (Grant 31200795), the Project of the Major Research Plan of the National Natural Science Foundation of China (Grant 91324201) and self-determined research funds of CCNU from the colleges' basic research and operation of MOE (CCNU14Z02015, CCNU14Z02004). Note that Xucheng Guo is now at the School of Public Management, Hunan Normal University. This work was carried out at Central China Normal University.

Received 31 January 2015; revision 17 July 2016; accepted 21 July 2016. growing body of research has shown that, in addition to features of the procedure (also called content information), an important source of information is the ease with which associative information is retrieved from memory, known as ease-of-retrieval or accessibility experiences (see, e.g., Greifeneder, Müller, Stahlberg, Van den Bos, \& Bless, 2011; Janssen, Müller, \& Greifeneder, 2011).

For social psychologists, these findings lead to an important question: under what conditions do people rely on ease-of-retrieval (vs. content information) to form procedural justice judgements? According to the uncertainty management model (Van den Bos \& Lind, 2002), procedural justice matters to people because it can reduce discomfort and anxiety induced by uncertainties, and forming procedural justice judgements helps people to manage uncertainties. Accordingly, uncertainty is a crucial moderator of procedural justice judgements. In support of this general hypothesis, research has demonstrated that salience of personal uncertainty moderates reliance on ease-of-retrieval in procedural justice judgements (see, e.g., Greifeneder et al., 2011; Janssen et al., 2011).

Here we argue that it is important to distinguish between two types of uncertainties: informational uncertainty and personal uncertainty (Van den Bos, 2001). Informational uncertainty involves having less information available than one ideally would like to have in order to be able to confidently form a given social judgement (Van den Bos \& 
Lind, 2009). Personal uncertainty is defined as a subjective sense of doubt or instability in self-views, worldviews, or the interrelation between the two (Van den Bos \& Lind, 2009). The two types of uncertainties are different in nature. Personal uncertainty is derived from the internal self, and is related to the motivational system (Van den Bos \& Lind, 2002; Van den Bos \& Lind, 2009). In contrast, the source of informational uncertainty is the environment and a lack of external information (Van den Bos, 2003). Hence internal personal uncertainty and external informational uncertainty have been considered to fulfill distinct functions in forming procedural justice judgements (Greifeneder et al., 2011).

Previous research examining ease-of-retrieval effects focused on personal uncertainty only (e.g., Greifeneder et al., 2011; Müller, Greifeneder, Stahlberg, Van den Bos, $\&$ Bless, 2010). We note that this research neglected another potentially important moderator - informational uncertainty. In the present research, therefore, we investigated whether reliance on ease-of-retrieval is particularly pronounced under conditions of informational uncertainty. Furthermore, we studied the joint effect of informational uncertainty and personal uncertainty on reliance on ease-of-retrieval in making procedural justice judgements.

\section{Procedural justice judgements based on ease-of-retrieval}

According to Tversky and Kahneman (1973), estimation of the frequency of an event is based on the ease of retrieving relevant instances or associations. When relevant exemplars are easy to bring to mind, a given class of events is judged to be frequent, whereas when exemplars are difficult to bring to mind the class is judged to be rare. To investigate whether reliance on ease-of-retrieval influences procedural justice judgements, researchers have used the ease-of-retrieval paradigm introduced by Schwarz et al. (1991) and asked participants to recall few or many unfair (or fair) aspects of procedural justice. Participants recalling few aspects felt this retrieval task to be easy, whereas participants recalling many aspects felt the retrieval task to be difficult (Greifeneder et al., 2011; Janssen et al., 2011; Müller et al., 2010). Importantly, if participants rely on content information when forming procedural justice judgements, they should judge the procedure as less fair just after retrieving many compared to few unfair aspects. In contrast, if participants rely on easeof-retrieval, they should evaluate the procedure as more fair just after retrieving many compared to few unfair aspects (Greifeneder et al., 2011). The results of these studies indeed showed that ease-of-retrieval can constitute an essential source of information when forming procedural justice judgements (Greifeneder et al., 2011; Janssen et al., 2011; Müller et al., 2010).

\section{Moderating effects of personal uncertainty}

Both ease-of-retrieval and content information are important sources of information in procedural justice judgements. It is important to understand, therefore, under what conditions people rely on one of the two kinds of information source. In examining this issue, Greifeneder et al. (2011) and Müller et al. (2010) focused on the moderating effect of personal uncertainty on the relationship between ease-of-retrieval and procedural justice judgements. For instance, Greifeneder et al. (2011) found that, in situations where personal certainty was salient, participants recalling many unfair procedural aspects made high procedural justice judgements, whereas when personal uncertainty was salient, participants recalling few unfair procedural aspects made high procedural justice judgements. These findings suggest that personal certainty individuals rely on ease-of-retrieval to form procedural justice judgements, whereas personal uncertainty individuals rely on content information.

This moderating effect of personal uncertainty is probably because personal uncertainty makes issues of procedural justice more self-relevant to people (De Cremer \& Sedikides, 2005). As self-relevance increases, people are motivated to process information systematically (Petty \& Cacioppo, 1986). It seems likely that salience of personal uncertainty may lead people to rely more strongly on content information, because they need this information to clarify the self. In contrast, when personal certainty is salient, the content information is of less self-relevance and hence under these conditions people may rely more strongly on ease-ofretrieval when forming procedural justice judgements (Greifeneder et al., 2011).

\section{Moderating effects of informational uncertainty}

Importantly, the findings of moderating effect of personal uncertainty on individuals' reliance on ease-of-retrieval seems to be different from what follows from research on informational uncertainty. After all, research has revealed that informational uncertainty results in a higher possibility that people will rely on heuristic cues to form judgements (e.g., Van den Bos, 2003; Van den Bos \& Lind, 2002). Greifeneder et al. (2011), and therefore assumed that the moderation effect of informational uncertainty on individuals' reliance on ease-of-retrieval may differ from effects obtained when personal uncertainty was the moderator. However, as of now, the moderation effect of informational uncertainty on the relationship between easeof-retrieval and procedural justice judgements has not been investigated. The current paper will fill this void. 
Furthermore, we predict that the reliance on easeof-retrieval is more pronounced under conditions of informational uncertainty (Van den Bos \& Lind, 2002). After all, in these information-uncertain situations individuals tend to rely on heuristic cues, like cognitive feelings, when forming their justice judgements (Greifeneder et al., 2011; Van den Bos, 2003), because ambiguous or uncertain messages would reduce subjects' processing ability (Petty \& Cacioppo, 1986).

Conversely, in information-certain situations, where the relevant information is available, individuals are likely to rely on the available content information to evaluate procedural justice. This is because when the message is unambiguous and certain, individuals have the ability to process content information systematically (Petty \& Cacioppo, 1986). In correspondence with this line of reasoning, Van den Bos (2003) reported that in information-uncertain conditions, due to insufficient information, individuals may rely on affect as a source of heuristic clues to make procedural justice judgements; in information-certain conditions, the characteristics of the procedure act as the source of judgements.

Based on this line of reasoning, we hypothesized that informational uncertainty may moderate individuals' reliance on ease-of-retrieval. Specifically, we proposed that individuals in information-uncertain conditions may rely on ease-of-retrieval whereas those in information-certain conditions are likely to rely on content information.

We also investigate how the interplay of informational uncertainty and personal uncertainty may affect the relationship between ease-of-retrieval and procedural justice judgements. We note that, when considering processing ability and motivation simultaneously, individuals tend to rely on content information under conditions of high processing ability and high motivation (Petty \& Cacioppo, 1986). In contrast, when either processing ability or motivation is low, judgements may be formed by heuristics, such as ease-of-retrieval (Petty \& Cacioppo, 1986). On the basis of this line of reasoning, we hypothesized that under conditions of personal uncertainty, informational certain individuals who possess both high processing ability and high motivation will rely on content information, whereas informational uncertain individuals lacking processing ability will rely on ease-of-retrieval processes. In contrast, under conditions of personal certainty, both informational uncertain and informational certain individuals are assumed to rely on ease-of-retrieval when forming procedural justice judgements due to their low processing motivation.

We conducted three studies to test our hypotheses. We first examined the predicted moderation effect of informational uncertainty in Study 1. In Study 2 we examined the underlying process of the moderation effect of informational uncertainty. We then examined the interaction of informational uncertainty and personal uncertainty as an influence on the effects of ease-of-retrieval on procedural justice judgements in Study 3.

\section{Study 1}

In Study 1 we examined whether informational uncertainty moderates reliance on ease-of-retrieval. Interviews before our experiments revealed that college students were sensitive to procedural justice in scholarship assessments, so we used that topic in our study. First, we manipulated informational uncertainty by varying two different types of 'no-voice' procedures (Van den Bos, 2003). The implicit-no-voice procedure did not inform participants about any possible opportunities to voice their opinions; hence, it implicitly did not allow them to voice opinions. In this procedure, participants lacked direct information about the procedure (Van den Bos \& Lind, 2002). The explicit-no-voice procedure informed participants about possible opportunities to voice their opinions, after which they were told that they would not get such an opportunity, hence explicitly denying them voice. In this procedure, participants had direct, explicit procedure information (Van den Bos, 2003). Importantly, the implicit-no-voice procedure led participants to respond to issues of procedural justice under conditions of informational uncertainty, whereas the explicit-no-voice procedure led them to react under conditions of informational certainty. Next, ease-of-retrieval was manipulated by the easeof-retrieval paradigm (Schwarz et al., 1991). Finally, the dependent variables were measured.

\section{Method}

Participants and design. A total of 80 Chinese college students ( 32 women, $M_{\text {age }}=19.38, S D=1.02$ ) from the same class participated in this experiment and were given a small reward for their participation. Participants were randomly assigned to one cell of a 2 (procedure: implicit-no-voice vs. explicit-no-voice) $\times 2$ (number of aspects: few vs. many) between-subjects design.

Procedure and materials. Participants were randomly assigned to one of two comparable rooms. The explicitno-voice procedure was conducted in one room, and the implicit-no-voice procedure was conducted in the other room. First, the experimenter told participants that 'in order to encourage students to develop in an all-around way, the college gave $300 \mathrm{CNY}$ to each class to reward the student who has good grades. The college will reward one student each month, and today we will elect this month's scholar. Because you are in the same class and you study together, you know each other very well, such as each other's grades, endeavours, and enthusiasm.' 
Next, participants in the explicit-no-voice condition were asked to think for one minute about who was worthy of 300 CNY. They were also told that there was a possibility that they would get an opportunity to voice their opinions about who should be the scholar. Several minutes later, the experimenter told them that he was just informed that the scholar had already been chosen, so the participants would not be asked to voice their opinions about who was worthy of $300 \mathrm{CNY}$. The participants in the implicit-no-voice condition were just asked to think for one minute about who was worthy of $300 \mathrm{CNY}$. Thus, neither condition was actually given the opportunity to voice their opinions, but this opportunity was explicitly denied in the explicit-no-voice condition and implicitly denied in the implicit-no-voice condition.

After the manipulation, the experimenter told the participants that they would participate in other unrelated studies. Participants were handed a pencil and a piece of paper that contained the instructions, ease-of-retrieval manipulation, and dependent measures. Ease-of-retrieval was manipulated by asking participants to retrieve unfair aspects of the election procedure. In the few condition, participants (19 who experienced the explicit-no-voice procedure and 21 who experienced the implicit-no-voice procedure) were asked to recall one unfair aspect of the procedure, whereas participants in the many condition (21 who experienced the explicit-no-voice procedure and 19 who experienced the implicit-no-voice procedure) were asked to recall six unfair aspects. An independent pre-test showed that recalling one unfair aspect of the election procedure was easy, whereas recalling six unfair aspects of the election procedure was difficult.

Dependent measures. The dependent variable was perceived procedural justice, which was assessed with three items - how just $(1=$ very unjust, $7=$ very just $)$, fair $(1=$ very unfair, $7=$ very fair $)$, and appropriate $(1=$ very inappropriate, $7=$ very appropriate $)$ - participants considered the election procedure that they had experienced. Participants' answers were averaged to form a reliable scale of procedural justice judgements (Cronbach's $\alpha=.86$ ).

Next, the manipulation checks were assessed. Two questions served as the manipulation check for easeof-retrieval: 'How easy or difficult was it for you to list unfair aspects of the election procedure?' and 'How easy or difficult would it have been for you to list more unfair aspects?' $(1=$ very difficult, $9=$ very easy; $r=.53, p<.01)$. Participants' answers to these two items were averaged. Two questions served as the manipulation check for the procedure. Participants were asked to what extent they agreed with the statements that they (i) had been explicitly informed that they would not receive an opportunity to voice their opinions about who should get the reward and (ii) had not been explicitly informed whether or not they would receive an opportunity to voice their opinions ( 1 = strongly disagree, 7 = strongly agree; Van den Bos, 2003).

\section{Results}

Manipulation checks. A 2 (procedure: implicit-no-voice vs. explicit-no-voice) $\times 2$ (number of aspects: few vs. many) analysis of variance (ANOVA) on the manipulation check for ease-of-retrieval only yielded a significant main effect of number of aspects, $F(1,76)=5.57, p<.05, \eta_{\mathrm{p}}^{2}=.07$. Participants who retrieved one unfair aspect of the election procedure rated the task easier $(M=5.18, S D=2.10)$ than those who retrieved six unfair aspects $(M=4.21, S D=1.74)$. These results indicate that the ease-of-retrieval manipulation was successful.

Two $2 \times 2$ ANOVAs on the two manipulation check questions for procedure yielded only a significant main effect of the procedure manipulation. For the explicit-no-voice check it was found that participants in the explicit-no-voice condition $(M=5.70, S D=1.38)$ agreed more with the statement that they had been explicitly informed that they would not receive an opportunity to voice their opinions than those in the implicit-no-voice condition $(M=3.58, S D=1.65)$, $F(1,76)=38.98, p<.001, \eta_{\mathrm{p}}^{2}=.34$. For the implicit-no-voice check it was found that participants in the implicit-no-voice condition $(M=4.78, S D=1.23)$ agreed more with the statement that they had not been explicitly informed whether or not they would receive an opportunity to voice their opinions than those in the explicit condition $(M=3.10$, $S D=1.79), F(1,76)=23.28, p<.001, \eta_{\mathrm{p}}^{2}=.23$. These results indicated that the procedure manipulation was perceived as intended.

Procedural justice. Procedural justice judgements were entered into a 2 (procedure: implicit-no-voice vs. explicitno-voice) $\times 2$ (number of aspects: few vs. many) ANOVA. Neither main effect was significant. As expected, there was a significant interaction between procedure and number of aspects, $F(1,76)=18.60, p<.001, \eta_{\mathrm{p}}^{2}=.20($ see Figure 1$)$. As predicted, simple main effects of the procedure manipulation within each of the number-of-aspects conditions indicated that participants in the implicit-no-voice condition rated the procedure as less just after retrieving few $(M=3.95, S D=0.75)$ rather than many unfair aspects $(M=4.68, S D=0.99), F(1,76)=4.76, p<.05, \eta_{\mathrm{p}}^{2}=.06$, reflecting reliance on ease-of-retrieval. In contrast, participants in the explicit-no-voice condition rated the procedure as more just after retrieving few $(M=4.05$, $S D=1.31)$ rather than many unfair aspects $(M=2.86$, $S D=0.90), F(1,76)=15.39, p<.001, \eta_{\mathrm{p}}^{2}=.17$, reflecting reliance on content information in forming their procedural justice judgements.

In addition, simple main effects of number of aspects within each of the two procedure conditions indicated that 


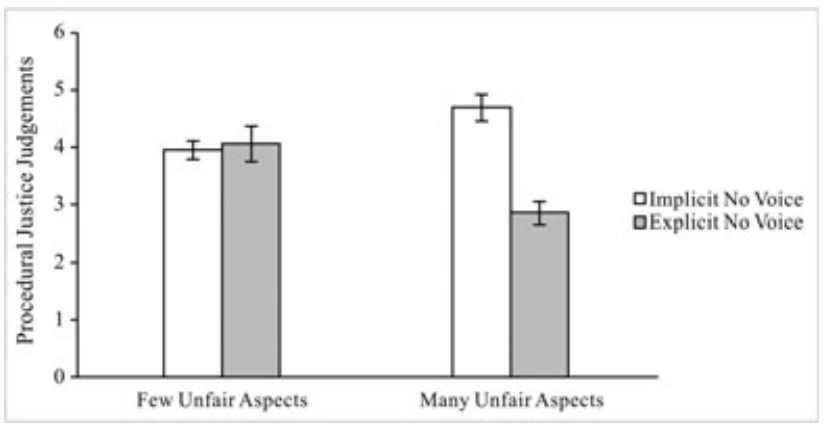

Figure 1 Procedural justice judgements as a function of number of aspects and procedure (Study 1). Means are on 7-point scales with higher scores indicating higher levels of procedural justice. Error bars represent standard errors of the mean.

procedural justice judgements did not differ between participants who recalled few unfair aspects and were in either the explicit- or implicit-no-voice conditions, $F(1,76)=.08, p>.05, \eta_{\mathrm{p}}^{2}=.00$. In contrast, when recalling many unfair aspects, participants in the implicitno-voice condition rated the procedure as more just than those in the explicit-no-voice condition, $F(1,76)=33.90$, $p<.001, \eta_{\mathrm{p}}^{2}=.31$.

\section{Discussion}

As expected, Study 1 revealed that individuals' reliance on ease-of-retrieval when forming procedural justice judgements is moderated by informational uncertainty. Specifically, individuals in information-uncertain conditions may rely on ease-of-retrieval, whereas those in informationcertain conditions may rely on content information. According to the results, the moderating effect of informational uncertainty is different from that of personal uncertainty. Given that previous research focused on the effect of personal uncertainty only, the present findings are particularly noteworthy.

\section{Study 2}

It should be noted that there were two limitations in Study 1. First, although Study 1 verified our hypothesis, there may be an alternative explanation for the results. Compared to the participants in the implicit-no-voice condition, participants in the explicit-no-voice condition had more material to recall (i.e., being told they would have a chance to voice their opinions, and later being told this was not the case), and thus it may have been easier for them to identify six unfair aspects. Second, Study 1 did not present data to support the claim that informational uncertainty increases reliance on heuristics because it lowers individuals' processing capacity rather than their differences in motivation. To compensate for these limitations, we conducted Study 2.

As in Study 1, Study 2 examined the effect of informational uncertainty on reliance on ease-of-retrieval in procedural justice judgements. The National Postgraduate Entrance Examination (NPEX) procedure was chosen as a research context for this study. Participants were master's students who passed the NPEX successfully two to three years ago. First, informational uncertainty about the NPEX procedure was assessed. Next, ease-of-retrieval was manipulated as in Study 1. Finally, process motivation, capacity were assessed.

\section{Method}

Participants and design. A total of 102 Chinese master's students (66 women, $M_{a g e}=23.84, S D=1.17$ ) participated in this experiment and were given a small reward for their participation. All participants received a booklet that contained the instructions, manipulation, and dependent measures. Using a between-subjects design, participants were randomly assigned to one of two conditions (number of aspects: few vs. many).

First, informational uncertainty was assessed by calculating the mean of two items: 'If I had more information about the procedure, I would have felt more certain about the National Postgraduate Entrance Examination procedure' and 'Missing information made me feel uncertain to assess the procedural justice' ( 1 = strongly disagree, $7=$ strongly agree; $r=.43, p<.01)$. Next, we asked participants to recall either one (few condition) or six (many condition) unfair aspects of the NPEX procedure.

Dependent measures. The dependent variable was perceived procedural justice, which was measured with the same three items as in Study 1. Again, participants' answers were averaged to form a reliable scale of procedural justice judgements (Cronbach's $\alpha=.81$ ). The manipulation check for the ease-of-retrieval manipulation $(r=.74, p<.01)$ was also the same as in Study 1. Next, two items assessed participants' motivation: 'I have high motivation to make accurate judgements' and 'I have put effort into making accurate judgements' $(r=.32, p<.01$; e.g., Greifeneder \& Bless, 2008). Items were rated on a 7-point Likert scale ( 1 = strongly disagree, $7=$ strongly agree). Two items assessed participants' perceptions of their processing capacity: 'I think I have enough capacity to make accurate judgements' and 'It is difficult for me to make accurate judgements' (reverse scored; $r=.35, p<.01$ ). Items were rated on a 7 -point Likert scale $(1=$ strongly disagree, 7 = strongly agree). 


\section{Results}

Manipulation check. The ease-of-retrieval index was standardized and entered as the dependent variable in a hierarchical regression analysis. Following Aiken and West (1991), informational uncertainty was standardized in all of the following analyses. The dummy-coded number of aspects $(0=$ few, $1=$ many $)$ and the continuous informational uncertainty score were entered as predictors in Step 1; the interaction between these two variables was entered in Step 2. Only the main effect of number of aspects was found to be statistically significant $(\beta=-.81, p<.001)$. The twoway interaction was not significant $(\beta=-.27, p>.05)$. These results indicated that participants who retrieved one unfair aspect of the NPEX procedure rated it easier $(M=5.17, S D=2.02)$ than those who retrieved six unfair aspects $(M=3.58, S D=1.76)$. Importantly, the nonsignificant two-way interaction indicated that ease or difficulty was unaffected by informational uncertainty.

Procedural justice. The procedural justice judgement scale was standardized and as the dependent variable in the described hierarchical regression analysis. The main effect of informational uncertainty was significant $(\beta=-.30$, $p<.05)$. Importantly, this analysis yielded the predicted significant interaction between number of aspects and informational uncertainty $\left(\beta=.48, p<.05\right.$; see Figure $\left.2^{1}\right)$. To further investigate this interaction, simple slopes were analyzed following the recommendations by Aiken and West (1991). Participants with high informational uncertainty (1 SD above the mean) reported higher procedural justice judgements after recalling many rather than few unfair aspects of the NPEX procedure $(\beta=.60, p<.05)$. This finding reflects reliance on ease-of-retrieval. In contrast, the simple slope for participants with low informational uncertainty (1 SD below the mean) was negative $(\beta=-.36$, $p>.05)$. The negative slope suggested that participants who recalled many (compared to few) unfair aspects tended to rate the NPEX procedure as more unfair.

Capacity and motivation. To test whether participants differed in their capacity rather than motivation, we established a series of regression analyses. Two independent regression analyses were conducted to examine the influence of informational uncertainty on standardized capacity and standardized motivation. The results revealed a significant negative association between informational uncertainty and capacity $(\beta=-.41, p<.001)$. Informational uncertainty was not associated with motivation $(\beta=.005, p>.05)$.

Next, the procedural justice judgement scale was standardized and entered as the dependent variable in a hierarchical regression analysis. Informational uncertainty was included as a covariate in Step 1. The standardized capacity and the dummy-coded number of aspects $(0=$ few, $1=$ many) were entered as predictors in Step 2. The interaction term was entered in Step 3. Indeed, a significant two-way interaction effect emerged $(\beta=-.45, p<.05)$. To further investigate this interaction, simple slopes were analyzed following the recommendations by Aiken and West (1991). For high processing capacity participants (1 SD above the mean), the simple slope was negative $(\beta=-.34$, $p>.05)$, which suggested that participants who recalled many (compared to few) unfair aspects tended to rate the NPEX procedure as more unfair. In contrast, low processing capacity participants (1 SD below the mean) reported higher procedural justice judgements after recalling many (compared to few) unfair aspects of the NPEX procedure $(\beta=.56, p=.05)$, which reflected reliance on easeof-retrieval.

\section{Discussion}

These findings suggest that the moderation effect of informational uncertainty on individuals' reliance on easeof-retrieval is due to processing capacity rather than

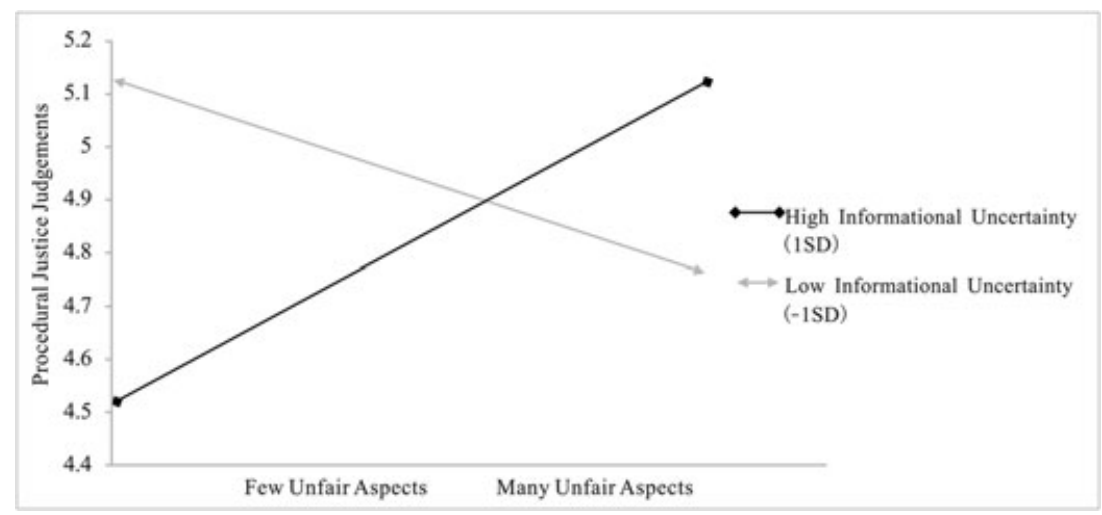

Figure 2 Procedural justice judgements as a function of number of aspects and informational uncertainty (Study 2). Means are on 7-point scales with higher scores indicating higher levels of procedural justice. 
motivation to process. Two aspects of these findings deserve mention. First, informational uncertainty was assessed rather than manipulated in Study 2. The results of the manipulation check for the number of aspects in Study 2 showed that only the main effect of number of aspects was statistically significant; therefore, it appears safe to suggest that participants' difference in ease-of-retrieval was not caused by informational uncertainty. Second, the obtained interaction between number of aspects and processing capacity on procedural justice judgements allows for conclusions about why individuals in conditions of informational uncertainty are likely to rely on ease-ofretrieval, which will be further discussed in the General discussion section.

\section{Study 3}

Study 3 examined the possible interaction effect of informational uncertainty and personal uncertainty on reliance on ease-of-retrieval in procedural justice judgements. First, procedure and ease-of-retrieval were manipulated as in Study 1. Next, salience of personal uncertainty was manipulated by varying whether our participants were asked to complete two open-ended questions about their emotions and physical sensations when they felt uncertain or certain about themselves (Van den Bos, 2001). In order to rule out effects of the personal uncertainty salience manipulation on the procedure manipulation and the quality of the generated unfair aspects (Greifeneder et al., 2011), the procedure and ease-ofretrieval were manipulated before the personal uncertainty salience manipulation.

\section{Method}

Participants and design. A total of 149 Chinese college students $\left(112\right.$ women, $\left.M_{\text {age }}=21.58, S D=1.71\right)$ participated in this experiment voluntarily and were given a small reward for their participation. All participants received a booklet that contained the instructions, manipulations, and dependent measures. Participants were randomly assigned to one cell of a 2 (procedure: implicit-no-voice vs. explicit-no-voice) $\times 2$ (salience: personal uncertainty salient vs. personal certainty salient) $\times 2$ (number of aspects: few vs. many) betweensubjects design.

Procedure and materials. All participants read the following scenario and were asked to imagine that they had recently experienced the situation:

Six students and you formed a team to take part in the 'scientific research project.' Because of your efforts, your school approved your team application. Afterwards, you demonstrated, experimented, and surveyed zealously.
Throughout the process you had an outstanding performance, which was obvious to all. When others could not solve a problem, you solved it through unremitting efforts. When others wanted to give up, you motivated them to continue. Finally, you not only completed the project successfully, but your project also won the first prize, and the school rewarded your team $3000 \mathrm{CNY}$. An assistant would allocate the money between you and your six team members.

Participants in the implicit-no-voice condition then read the following:

The assistant called you all and asked you to think about how to allocate the money. You all thought about it seriously, and the assistant told you that he would do a related survey first and then allocate the $3000 \mathrm{CNY}$.

Participants in the explicit-no-voice condition read the following:

The assistant called you all and asked you to think about how to allocate the $3000 \mathrm{CNY}$. You were informed that there was a possibility that you could get an opportunity to voice your opinions about how to allocate the 3000 CNY. You all thought about the methods seriously, and at this time the assistant suddenly told you that the department leaders had already made a decision, so you would not be asked to voice your opinions about how to allocate the $3000 \mathrm{CNY}$.

When the participants reported that they had finished imagining the scenario, the experimenter collected the scenario paper and asked the participants to recall the unfair aspects. As in Study 1, the participants were asked to recall either one or six unfair aspects about the procedure they just imagined. Next, personal (un)certainty salience was manipulated. Participants in the personal uncertainty salient condition were asked to remember and vividly recall a situation in which they felt uncertain about themselves and to write down their answers to the following two questions: (1) 'Please briefly describe the emotions that the thought of your being uncertain arouses in you;' and (2) 'Please write down what you think will physically happen to you as you feel uncertain' (Van den Bos, 2001). In the condition where personal certainty was salient, the instructions and questions asked were the same but the word 'uncertain' was replaced by 'certain' (Van den Bos, 2001).

Dependent measures. The dependent variable was perceived procedural justice, which was measured with the same three items as in Study 1. Again, participants' answers were averaged to form a reliable scale of procedural justice judgements (Cronbach's $\alpha=.81$ ). The manipulation checks for the ease-of-retrieval manipulation $(r=.67, p<.01)$ and the procedure manipulation were also the same as in Study 1. 


\section{Results}

Manipulation checks. A 2 (procedure: implicit-no-voice vs. explicit-no-voice) $\times 2$ (salience: personal uncertainty salient vs. personal certainty salient) $\times 2$ (number of aspects: few vs. many) ANOVA on ease or difficulty ratings yielded only a significant main effect of number of aspects. Participants who retrieved one unfair aspect felt it easier to retrieve unfair aspects of the money distributing procedure $(M=6.36$, $S D=1.46)$ than those retrieving six unfair aspects $(M=3.01, S D=1.47), F(1,141)=198.20, p<.001, \eta_{\mathrm{p}}^{2}=.58$. These results indicated that the ease-of-retrieval manipulation was successful.

Two $2 \times 2 \times 2$ ANOVAs on the two manipulation check questions for procedure showed only a significant main effect of the procedure manipulation. For the explicit-no-voice manipulation check, participants in the explicit-no-voice condition $(M=5.95, S D=1.25)$ agreed more with the statement that they had been explicitly informed that they would not receive an opportunity to voice their opinions than those in implicit-no-voice condition $(M=3.74, S D=1.63)$, $F(1,141)=85.63, p<.001, \eta_{\mathrm{p}}^{2}=.38$. For the implicitno-voice manipulation check, participants in the implicitno-voice condition $(M=4.08, S D=1.58)$ agreed more with the statement that they had not been explicitly informed whether or not they would receive an opportunity to voice their opinions than those in the explicit condition $(M=2.90, S D=1.54), F(1,141)=21.73, p<.001, \eta_{\mathrm{p}}^{2}=.13$. These results indicated that the procedure manipulation was successful.

Following Van den Bos (2001), two judges checked what participants wrote down during the manipulation. The two judges agreed (with $100 \%$ agreement) that the participants in the personal uncertainty salient condition had been thinking about their being personally uncertain, whereas the participants in the personal certainty salient condition had been thinking about their being personally certain.

Procedural justice. A 2 (procedure: implicit-no-voice vs. explicit-no-voice) $\times 2$ (salience: personal uncertainty salient vs. personal certainty salient) $\times 2$ (number of aspects: few vs. many) ANOVA on the procedural justice judgements yielded a significant main effect of procedure, $F(1,141)=25.49, p<.001, \eta_{\mathrm{p}}^{2}=.15$, a twoway interaction between procedure and number of aspects, $F(1,141)=5.52, p<.05, \eta_{\mathrm{p}}^{2}=.04$, and a two-way interaction between personal uncertainty salience and number of aspects, $F(1,141)=14.10, p<.01, \eta_{\mathrm{p}}^{2}=.09$. Importantly, these effects were qualified by the hypothesized three-way interaction between procedure, number of aspects, and salience, $F(1,141)=5.31, p<.05, \eta_{\mathrm{p}}^{2}=.04$ (see Figure 3).

The significant three-way interaction effect indicated that the two-way interaction between procedure and number of

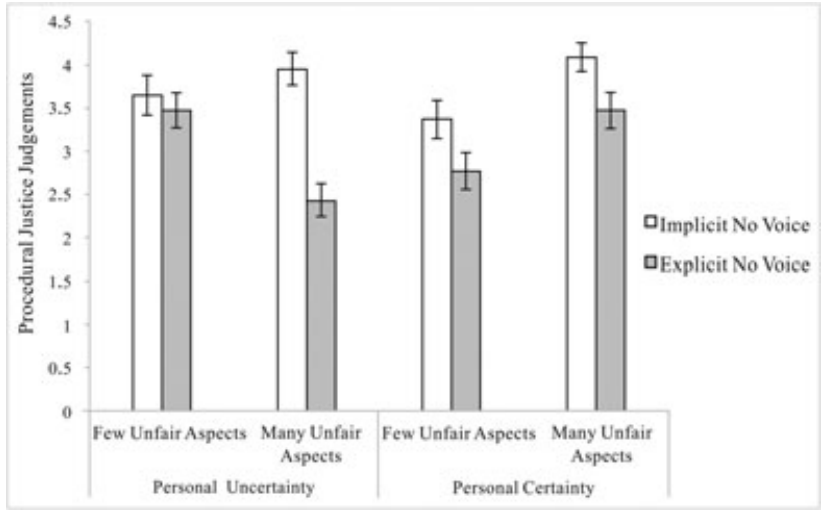

Figure 3 Procedural justice judgements as a function of procedure, number of aspects, and personal uncertainty salience (Study 3). Means are on 7-point scales with higher scores indicating higher levels of procedural justice. Error bars represent standard errors of the mean.

aspects was qualified by whether participants were in the personal uncertainty salience condition or in the personal certainty salience condition. Indeed, testing for appropriate simple interaction effects indicated that the two-way interaction between procedure and number of unfair aspects was not significant in the personal certainty salience condition, $F(1,146)=0.04, p>.05, \eta_{\mathrm{p}}^{2}=.00$, but was significant in the personal uncertainty salience condition, $F(1,146)=9.99, p<.01, \eta_{\mathrm{p}}^{2}=.06$. In the personal certainty salience condition, participants rated the procedure as more just after retrieving many $(M=3.80, S D=0.83)$ rather than few unfair aspects $(M=3.08, S D=0.97), F(1,68)=12.31$, $p<.01, \eta_{\mathrm{p}}^{2}=.15$. Perhaps more interestingly, participants in the personal uncertainty salience condition, after having received the explicit-no-voice manipulation, evaluated the procedure as more just after retrieving few $(M=3.47$, $S D=0.90)$ rather than many aspects $(M=2.43, S D=0.85)$, $F(1,146)=11.61, p<.01, \eta_{\mathrm{p}}^{2}=.07$, reflecting reliance on content information. In contrast, participants in the personal uncertainty salience condition, after having received the implicit-no-voice manipulation, evaluated the procedure as somewhat more unjust after retrieving few $(M=3.65$, $S D=0.99)$ rather than many aspects $(M=3.95, S D=0.84)$, $F(1,146)=1.18, p>.05, \eta_{\mathrm{p}}^{2}=.008$, reflecting a tendency of reliance on ease-of-retrieval, although the difference was not significant.

\section{Discussion}

Consistent with our hypotheses, Study 3 suggests that, under conditions of personal uncertainty, informational certain individuals rely on content information when forming procedural justice judgements, whereas informational uncertain individuals showed a tendency to rely on ease- 
of-retrieval processes. In contrast, under conditions of personal certainty, both informational uncertain and informational certain individuals rely on ease-of-retrieval when forming procedural justice judgements. The results suggest that the joint effect of personal and informational uncertainty on reliance on ease-of-retrieval is different from the two uncertainties acting alone. And this will be further discussed in the General Discussion section.

We noted that in the conditions where personal uncertainty was salient, the ease-of-retrieval effect was less pronounced among informational uncertain participants. This may be due to the strong effects that personal uncertainty salience can have on people (Van den Bos \& Lind, 2002; Van den Bos \& Lind, 2009). When personal uncertainty was made salient in our research by means of experimental manipulation, this may have led them to be highly motivated to process information and they may have been oriented to rely strongly on content information. As a result, the potential ease-of-retrieval effects were attenuated among conditions where personal uncertainty was salient. Further research is needed to back up this explanation of this aspect of our findings. This noted, if the explanation would turn out to have merit this could be a potentially important implication of our current findings, also because our results show that at least sometimes individuals may rely on ease-of-retrieval to form justice judgements under conditions where the relevant information is uncertain.

\section{General discussion}

Previous research on potential moderators of easeof-retrieval reliance focused on personal uncertainty only (e.g., Greifeneder et al., 2011; Janssen et al., 2011), but the moderation effect of informational uncertainty on individuals' reliance on ease-of-retrieval may differ from effects obtained when personal uncertainty was the moderator (Greifeneder et al., 2011). However, we are not aware of any prior empirical substantiation of this possibility. Hence, the present research was designed to fill this gap. We first investigated how informational uncertainty moderates individuals' reliance on ease-of-retrieval. The findings of Study 1 showed that individuals' reliance on ease-of-retrieval when forming procedural justice judgements is moderated by informational uncertainty. The findings of Study 2 revealed that the moderation effect of informational uncertainty on individuals' reliance on ease-of-retrieval is due to capacity rather than motivation to process. Study 3 explored the joint effect of informational uncertainty and personal uncertainty on reliance on ease-of-retrieval. Study 3 revealed that personal certain individuals (both informational uncertainty and informational certainty) rely on ease-of-retrieval to form procedural justice judgements. Personal uncertain individuals who felt informational certainty relied on content information, whereas those who felt informational uncertainty showed a tendency to rely on ease-of-retrieval.

The present study contributes to work on the uncertainty management model. According to this model, informational uncertainty plays a pivotal role in the procedural justice judgements process, whereas personal uncertainty is related to self-regulation processes (Van den Bos, 2007; Van den Bos \& Lind, 2009). Hence, informational uncertainty and personal uncertainty were studied independently in earlier studies. When studying the process of forming procedural justice judgements, researchers have focused more on informational uncertainty (e.g., Van den Bos, 2003). However, when studying uncertainty in reactions to procedural justice, many researchers have focused on personal uncertainty (e.g., De Cremer \& Sedikides, 2005). Our research used an approach combining the two uncertainties to study the process of forming procedural justice judgements. Previous studies used justice substitutability effects, which are proposed by the uncertainty management model, to explain how individuals form justice judgements under conditions of uncertainty (e.g., Van den Bos, 2003). In line with the justice substitutability notion, under conditions of information uncertainty, people may use other unrelated information, such as affect, to form procedural justice judgements (Van den Bos, 2003; Van den Bos \& Lind, 2002). However, the justice substitutability effects, which focus on informational uncertainty only, are of limited use when trying to explain the effects of the joint effect of informational uncertainty and personal uncertainty on forming procedural justice judgements, because the justice substitutability effects cannot explain the effect of motivation-related individual factors, such as personal uncertainty. In order to develop a more complete justice and uncertainty model, future research may focus on both informational uncertainty and personal uncertainty when exploring the relationship between uncertainty and justice judgements. This approach enables us to combine processing motivation with processing capability to interpret the joint effect.

In addition to testing our primary hypothesis, Study 2 was conducted to shed light on the processes underlying easeof-retrieval judgements. The results of Study 2 revealed that information uncertainty increases reliance on heuristics because it lowers people's processing ability. These findings help to bridge the literatures of justice and social cognition. This connection is important because researchers have emphasized the importance of investigating the cognitive mechanism underlying procedural justice judgements (Van den Bos, 2003; Van den Bos \& Lind, 2002).

Our results have practical implications as well, for example, with regard to employees' perceptions of organizational justice during periods of organizational change. In these circumstances of organizational turmoil where there often is a lot of personal and informational uncertainty among employees, managers are often faced with 
the task of deciding about important issues and the way in which they do this may cause some employees to experience procedural unfairness. In order to elicit and maintain employees' favourable work attitudes and behaviours in these periods, managers perhaps can make use of the easeof-retrieval insights studied in this paper. Ease-of-retrieval strategy constitutes one of the less costly strategies for improving employees' procedural justice judgements under conditions of both personal and informational uncertainty. Furthermore, we emphasize explicitly that using heuristic strategies should depend on organization circumstances and that managers should do their utmost best to ensure procedurally fair conditions and lead employees to form accurate justice judgements by suppressing possible biases caused by heuristic strategies. This would make it possible for employees to manage their uncertainty concerns by means of fair management practices.

In addition, some other important issues about the present research must be discussed. First, readers may find that the results in Studies 1 and 2 were similar as the results of the personal-uncertain conditions in Study 3. According to the characteristic of participants in Studies 1 (undergraduates) and 2 (master's degree students), we can speculate that participants were in a state of personal uncertainty to some extent. Especially for the participants in Study 2, these master's students were also asked to evaluate their life satisfaction by means of one item: 'I'm satisfied with my postgraduate life' $(1=$ strongly disagree, $5=$ strongly agree). The result showed that their life satisfaction was generally low $(M=2.63, S D=0.77)$. After interviewing these students, we found that their low life satisfaction was mainly caused by their uncertainty about their selfvalues. When studying one type of uncertainty's moderating effect, the other type may by default need to be considered as well, with a focus on two kinds of uncertainties simultaneously.

The second issue pertains to the reliability and validity of our findings. Readers might might wonder whether affective states provide an alternative explanation for the findings reported. With respect to informational uncertainty, we note that Van den Bos (2003) reported that there was no significant difference in PANAS ratings between participants in the explicit-no-voice and those in the implicit-no-voice conditions, making it not very likely that affective states provide an alternative explanation of the findings reported. Furthermore, we used different methods to operationalize informational uncertainty, which was manipulated in terms of explicit/implicit-no-voice procedure in Study 1 and was measured as well in Study 2. Study 2 replicated the findings of Study 1, thus supporting the reliability of the findings. With respect to personal uncertainty, the previous study has shown that the manipulation of personal uncertainty salience did not engender the positive and negative affect (Van den Bos,
Poortvliet, Maas, Miedema, \& Van den Ham, 2005). In this study, the PANAS was administered immediately following the personal uncertainty-salience manipulation. And results have all shown that participants' scores on the PANAS subsets yielded no significant effects (Van den Bos et al., 2005). This suggests that affect cannot explain the findings reported here.

Finally, we noted that all of our samples were from China. Given that the using of general heuristics (e.g., availability heuristics) is relatively universal across cultures (Nisbett \& Ross, 1980), the way individuals' use heuristics to form procedural justice judgements may be consistent across different cultures. However, the magnitude of heuristicrelated effects on justice judgements may differ across cultures (Qin, Ren, Zhang, \& Johnson, 2015). For instance, cultures differ in uncertainty avoidance (Hofstede, Hofstede, \& Minkov, 2010), which may moderate the magnitude of reliance on decision heuristics. Specifically, strong uncertainty avoidance cultures may be more likely to rely on decision heuristics (Jung \& Kellaris, 2004). Interestingly, it has been suggested that China is a weak uncertainty avoidance culture (Hofstede et al., 2010), which would imply that the degree of people's reliance on decision heuristics in information-uncertain conditions may be less than those in strong uncertainty avoidance cultures. Perhaps future research will show that the uncertainty avoidance moderates the effects of ease-of-retrieval on procedural justice judgements.

\section{Conclusions}

Although research has shown that personal uncertainty is a moderator of the ease-of-retrieval reliance in procedural justice judgements, relatively little is known about how another type of uncertainty-informational uncertaintyaffects ease-of-retrieval reliance. We demonstrated that informational uncertainty is a critical determinant of the source of information individuals rely on. Furthermore, our findings reveal that the moderating mechanism of informational uncertainty is different from personal uncertainty. Moreover, we also showed how the combined effect of personal and informational uncertainty may impact people's ease-of-retrieval reliance. These findings fit our integrative view of the work on judgements under informational uncertainty and the literature on personal uncertainty.

\section{Endnote}

1. For ease of interpretation and to make Figure 2 comparable with Figures 1 and 3, Figure 2 was drawn based on the results with the unstandardized procedural justice judgments scores. 


\section{References}

Aiken, L. S., \& West, S. G. (1991). Multiple regression: Testing and interpreting interactions. Newbury Park, CA: Sage.

Ambrose, M. L., \& Schminke, M. (2009). The role of overall justice judgments in organizational justice research: A test of mediation. Journal of Applied Psychology, 94, 491. doi: 10.1037/a0013203

De Cremer, D., \& Sedikides, C. (2005). Selfuncertainty and responsiveness to procedural justice. Journal of Experimental Social Psychology, 41, 157-173. doi: 10.1016/j. jesp.2004.06.010

Greifeneder, R., \& Bless, H. (2008). Depression and reliance on ease-of-retrieval experiences. European Journal of Social Psychology, 38, 213-230. doi: 10.1002/ejsp.451

Greifeneder, R., Müller, P. A., Stahlberg, D., Van den Bos, K., \& Bless, H. (2011). Beyond procedure's content: The role of accessibility experiences and personal uncertainty in procedural justice judgments. Experimental Psychology, 58, 341-352. doi: 10.1027/ 1618-3169/a000101

Hofstede, G., Hofstede, G. J., \& Minkov, M. (2010). Cultures and organizations: Software of the mind (3rd ed.). New York, NY: McGraw-Hill.

Janssen, J., Müller, P., \& Greifeneder, R. (2011). Cognitive process in procedural justice judgment: The role of ease-of-retrieval, uncertainty and experience. Journal of Organizational Behavior, 32, 726-750. doi: 10.1002/job.700

Jung, J. M., \& Kellaris, J. J. (2004). Crossnational differences in proneness to scarcity effects: The moderating roles of familiarity, uncertainty avoidance, and need for cognitive closure. Psychology and Marketing, 21(9), 739-753. doi: 10.1002/mar.20027

Müller, P. A., Greifeneder, R., Stahlberg, D., Van den Bos, K., \& Bless, H. (2010). Shaping cooperation behavior: The role of accessibility experiences and uncertainty. European Journal of Social Psychology, 40, 178-187. doi: 10.1002/ejsp.632

Nisbett, R. E., \& Ross, L. (1980). Human inference: Strategies and shortcomings of social judgment. Englewood Cliffs, NJ: Prentice-Hall.

Petty, R. E., \& Cacioppo, J. T. (1986). The elaboration likelihood model of persuasion. In L. Berkowiz (Ed.), Advances in experimental social psychology. New York: Academic Press.

Qin, X., Ren, R., Zhang, Z. X., \& Johnson, R. E. (2015). Fairness heuristics and substitutability effects: Inferring the fairness of outcomes, procedures, and interpersonal treatment when employees lack clear information. Journal of Applied Psychology, 100(3), 749-66. doi: 10.1037/a0038084

Schwarz, N., Bless, H., Strack, F., Klumpp, G., Rittenauer-Schatka, H., \& Simons, A. (1991). Ease of retrieval as information: Another look at the availability heuristic. Journal of Personality and Social Psychology, 61, 195-202. doi: 10.1037/00223514.61.2.195

Tversky, A., \& Kahneman, D. (1973). Availability: A heuristic for judging frequency and probability. Cognitive Psychology, 5, 207-232. doi: 10.1016/0010-0285(73)900339

Tyler, T. R. (2006). Psychological perspectives on legitimacy and legitimation. Annual Review of Psychology, 57, 375-400. doi: 10.1146/annurev.psych.57.102904.190038
Van den Bos, K. (2001). Uncertainty management: The influence of uncertainty salience on reactions to perceived procedural fairness. Journal of Personality and Social Psychology, 80, 931-941. doi: 10.1037/ 0022-3514.80.6.931

Van den Bos, K. (2003). On the subjective quality of social justice: The role of affect as information in the psychology of justice judgments. Journal of Personality and Social Psychology, 85, 482-498. doi: 10.1037/ 0022-3514.85.3.482

Van den Bos, K. (2007). Hot cognition and social justice judgments: The combined influence of cognitive and affective factors on the justice judgment process. In D. De Cremer (Ed.), Advances in the psychology of justice and affect (pp. 59-82). Greenwich, CT: Information Age Publishing.

Van den Bos, K., \& Lind, E. A. (2002). Uncertainty management by means of fairness judgments. In M. P. Zanna (Ed.), Advances in experimental social psychology (pp. 1-60). San Diego, CA: Academic Press.

Van den Bos, K., \& Lind, E. A. (2009). The social psychology of fairness and the regulation of personal uncertainty. In R. M. Arkin, K. C. Oleson, \& P. J. Carroll (Eds.), Handbook of the uncertain self (pp. 122-141). New York, NY: Psychology Press.

Van den Bos, K., Poortvliet, P. M., Maas, M., Miedema, J., \& Van den Ham, E. J. (2005). An enquiry concerning the principles of cultural norms and values: The impact of uncertainty and mortality salience on reactions to violations and bolstering of cultural worldviews. Journal of Experimental Social Psychology, 41, 91-113. doi: 10.1016/j. jesp.2004.06.001 